Article

\title{
Secure Total Domination in Rooted Product Graphs
}

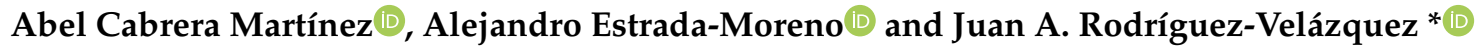 \\ Departament d'Enginyeria Informàtica i Matemàtiques, Universitat Rovira i Virgili, Av. Països Catalans 26, \\ 43007 Tarragona, Spain; abel.cabrera@urv.cat (A.C.M.); alejandro.estrada@urv.cat (A.E.-M.) \\ * Correspondence: juanalberto.rodriguez@urv.cat
}

Received: 16 March 2020; Accepted: 14 April 2020; Published: 15 April 2020

\begin{abstract}
In this article, we obtain general bounds and closed formulas for the secure total domination number of rooted product graphs. The results are expressed in terms of parameters of the factor graphs involved in the rooted product.
\end{abstract}

Keywords: secure total domination; total domination; domination; rooted product graph

\section{Introduction}

Recently, many authors have considered the following approach to the problem of protecting a graph [1-7]: suppose that one "entity" is stationed at some of the vertices of a (simple) graph $G$ and that an entity at a vertex can deal with a problem at any vertex in its closed neighbourhood. In general, an entity could consist of a robot, an observer, a legion, a guard, and so on. Informally, we say that a graph $G$ is protected under a given placement of entities if there exists at least one entity available to handle a problem at any vertex. Various strategies (or rules for entities placements) have been considered, under each of which the graph is deemed protected. As we can expect, the minimum number of entities required for protection under each strategy is of interest. Among these strategies we cite, for instance, domination [8,9], total domination [10], secure domination [1], secure total domination [2], Roman domination [6,7], Italian domination, [11] and weak Roman domination [5]. The first four strategies are described below.

The simplest strategies of graph protection are the strategy of domination and the strategy of total domination. In such cases, the sets of vertices containing the entities are dominating sets and total dominating sets, respectively. Typically, a vertex in a graph $G=(V(G), E(G))$ dominates itself and its neighbouring vertices. A set $S \subseteq V(G)$ is said to be a dominating set of $G$ if every vertex in $V(G) \backslash S$ is dominated by at least one vertex in $S$, while $S$ is said to be a total dominating set if every vertex $v \in V(G)$ is dominated by at least one vertex in $S \backslash\{v\}$.

The minimum cardinality among all dominating sets of $G$ is the domination number of $G$, denoted by $\gamma(G)$. The total domination number, denoted by $\gamma_{t}(G)$, is defined by analogy. These two parameters have been extensively studied. For instance, we cite the following books, [8-10].

Let $N(v)$ be the open neighbourhood of $v \in V(G)$ and let $S \subseteq V(G)$. In the case of the secure (total) domination strategy, a vertex $v \in V(G) \backslash S$ is deemed (totally) protected under $S \subseteq V(G)$ if $S$ is a (total) dominating set and there exists $u \in N(v) \cap S$ such that $(S \cup\{v\}) \backslash\{u\}$ is a (total) dominating set. In such a case, in order to emphasise the role of vertex $u$, we say that $v$ is (totally) protected by $u$ under $S$. A set $S \subseteq V(G)$ is said to be a secure (total) dominating set if every vertex in $v \in V(G) \backslash S$ is (totally) protected under $S$.

For instance, let $G$ be the graph shown in Figure 1, and suppose that an observer is stationed at vertex $a$ and another one is stationed at $b$. In such a case, the graph is under the control of the observers, as its vertices are (i.e., $\{a, b\}$ is a dominating set). Now, if the observer stationed at vertex $a$ moves to any vertex in $\{c, d, e\}$, then the graph is under the control of the observers as well. In this 
case, $\{a, b\}$ is a secure dominating set. Furthermore, if there are three observers and they are stationed at $a, b$, and $c$, then every vertex of the graph (including $a, b$, and $c$ ) is under the control of the observers, and this property is preserved if the observer stationed at $c$ moves to $d$ or $e$. Hence, $\{a, b, c\}$ is a secure total dominating set.

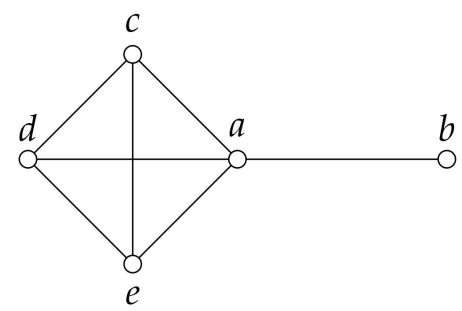

Figure 1. In this case, $\{a\}$ is a dominating set, $\{a, b\}$ is a total dominating set and also a secure dominating set, while $\{a, b, c\}$ is a secure total dominating set.

The minimum cardinality among all secure dominating sets of $G$ is the secure domination number of $G$, denoted by $\gamma_{s}(G)$. This domination parameter was introduced by Cockayne et al. in [1] and studied further in a number of works including [12-17]. Now, the minimum cardinality among all secure total dominating sets of $G$ is the secure total domination number of $G$, which is denoted by $\gamma_{s t}(G)$. This parameter was introduced by Benecke et al. in [2] and studied further in [3,4,16,18,19].

A secure total dominating set of cardinality $\gamma_{s t}(G)$ will be called a $\gamma_{s t}(G)$-set. A similar agreement will be assumed when referring to optimal sets associated to other parameters used in the article.

The problem of computing $\gamma_{s t}(G)$ is NP-hard [18], even when restricted to chordal bipartite graphs, planar bipartite graphs with arbitrary large girth and maximum degree three, split graphs and graphs of separability at most two. This suggests finding the secure total domination number for special classes of graphs or obtaining tight bounds on this invariant. This is precisely the aim of this article in which we study the case of rooted product graphs.

\section{Some Notation and Tools}

All graphs considered in this paper are finite and undirected, without loops or multiple edges. The minimum degree of a graph $G$ will be denoted by $\delta(G)$, i.e., $\delta(G)=\min _{v \in V(G)}|N(v)|$. As usual, the closed neighbourhood of a vertex $v \in V(G)$ is denoted by $N[v]=N(v) \cup\{v\}$. We say that a vertex $v \in V(G)$ is a universal vertex if $N[v]=V(G)$. By analogy with the notation used for vertices, the open neighbourhood of $S \subseteq V(G)$ is the set $N(S)=\cup_{v \in S} N(v)$, while the closed neighbourhood is the set $N[S]=N(S) \cup S$.

A set $S \subseteq V(G)$ is a double dominating set of $G$ if $|N[u] \cap S| \geq 2$ for every $u \in V(G)$. The double domination number of $G$, denoted by $\gamma_{\times 2}(G)$, is the minimum cardinality among all double dominating sets of $G$. The $k$-domination number of a graph $G$, denoted by $\gamma_{k}(G)$, is the cardinality of a smallest set of vertices such that every vertex not in the set is adjacent to at least $k$ vertices of the set. Such sets are called $k$-dominating sets.

Remark 1. Every secure total dominating set is a double dominating set and every double dominating set is a 2-dominating set. Therefore, for any graph $G$ with no isolated vertex, $\gamma_{s t}(G) \geq \gamma_{\times 2}(G) \geq \gamma_{2}(G)$.

By Remark 1, for every secure total dominating set $S$ and every vertex $v \in S$, the set $S \backslash\{v\}$ is a dominating set. Therefore, the following remark holds.

Remark 2. For every graph $G$ with no isolated vertex, $\gamma_{s t}(G) \geq \gamma(G)+1$.

A leaf of $G$ is a vertex of degree one. A support vertex of $G$ is a vertex which is adjacent to a leaf and a strong support vertex is a support vertex which is adjacent to at least two leaves. A leaf is said to be a 
strong leaf if it is adjacent to a strong support vertex, otherwise it is called a weak leaf. The set of leaves, support vertices, strong leaves and weak leaves are denoted by $\mathcal{L}(G), \mathcal{S}(G), \mathcal{L}_{\mathcal{S}}(G)$, and $\mathcal{L}_{w}(G)$, respectively.

Remark 3. If $D$ is a secure total dominating set of a graph $G$, then $(\mathcal{S}(G) \cup \mathcal{L}(G)) \subseteq D$ and no vertex of $G$ is totally protected under $D$ by vertices in $\mathcal{S}(G) \cup \mathcal{L}(G)$.

If $v$ is a vertex of a graph $H$, then the vertex-deletion subgraph $H-\{v\}$ is the subgraph of $H$ induced by $V(H) \backslash\{v\}$. In Section 3 we will show the importance of $\gamma_{s t}(H-\{v\})$ in the study of the secure total domination number of rooted product graphs. Now we proceed to state some basic tools.

Lemma 1. Let $H$ be a graph with no isolated vertex. If $v \in V(H) \backslash\left(\mathcal{L}_{w}(H) \cup \mathcal{S}(H)\right)$, then

$$
\gamma_{s t}(H-\{v\}) \geq \gamma_{s t}(H)-2
$$

Furthermore, if $\gamma_{s t}(H-\{v\})>\gamma_{s t}(H)$, then v belongs to every $\gamma_{s t}(H)$-set.

Proof. Assume that $v \in V(H) \backslash\left(\mathcal{L}_{w}(H) \cup \mathcal{S}(H)\right)$ and let $D$ be a $\gamma_{s t}(H-\{v\})$-set. Suppose that $|D| \leq \gamma_{s t}(H)-3$. If $|N(v) \cap D| \geq 2$, then $D \cup\{v\}$ is a secure total dominating set of $H$ of cardinality $|D \cup\{v\}| \leq \gamma_{s t}(H)-2$, which is a contradiction. Suppose that $|N(v) \cap D| \leq 1$. If $v \notin \mathcal{L}(H)$, then for every $y \in N(v) \backslash D$ we have that $D \cup\{v, y\}$ is a secure total dominating set of $H$ of cardinality $|D \cup\{v, y\}| \leq \gamma_{s t}(H)-1$, which is a contradiction. Now, if $v \in \mathcal{L}_{s}(H)$, then by Remark 3 we can conclude that $D \cup\{v\}$ is a secure total dominating set of $H$ of cardinality $|D \cup\{v\}| \leq \gamma_{s t}(H)-2$, which is a contradiction again. Hence, $\gamma_{s t}(H-\{v\})=|D| \geq \gamma_{s t}(H)-2$.

On the other hand, if there exists a $\gamma_{s t}(H)$-set $S$ such that $v \notin S$, then $S$ is a secure total dominating set of $H-\{v\}$, and so $\gamma_{s t}(H-\{v\}) \leq|S|=\gamma_{s t}(H)$. Therefore, if $\gamma_{s t}(H-\{v\})>\gamma_{s t}(H)$, then $v \in S$ for every $\gamma_{s t}(H)$-set $S$.

If $v$ is a weak leaf of $H$, then it could be that $\gamma_{s t}(H) \geq \gamma_{s t}(H-\{v\})+2$. For instance, Figure 2 shows the existence of cases in which the gap $\gamma_{s t}(H)-\gamma_{s t}(H-\{v\})$ is arbitrarily large. In Remark 4 we highlight this fact.

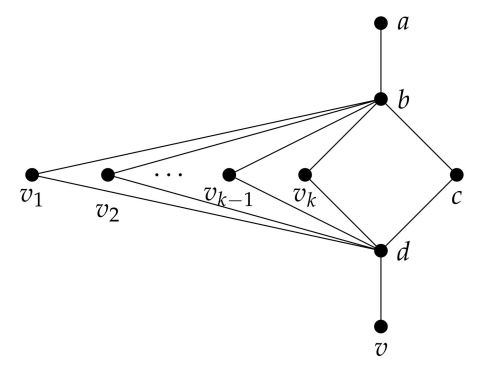

Figure 2. A graph $H$ where $V(H)$ is the $\gamma_{s t}(H)$-set. Since $\{a, b, c, d\}$ forms a $\gamma_{s t}(H-\{v\})$-set, we have that $\gamma_{s t}(H)-\gamma_{s t}(H-\{v\})=k+1$ for every integer $k \geq 1$.

Remark 4. For any integer $k \geq 1$ there exists a graph $H$ having a weak leaf vertex $v$ such that $\gamma_{s t}(H)-$ $\gamma_{s t}(H-\{v\})=k+1$.

In contrast to Remark 4, the following result shows the case where $v$ is a strong leaf.

Lemma 2. Let $H$ be a graph with no isolated vertex. If $v \in \mathcal{L}_{s}(H)$, then

$$
\gamma_{s t}(H-\{v\})=\gamma_{s t}(H)-1
$$

Proof. Let $D$ be a $\gamma_{s t}(H)$-set, $v \in \mathcal{L}_{s}(H)$ and $N(v)=\left\{s_{v}\right\}$. By Remark 3 we deduce that $D \backslash\{v\}$ is a secure total dominating set of $H-\{v\}$ and so $\gamma_{s t}(H-\{v\}) \leq|D \backslash\{v\}| \leq \gamma_{s t}(H)-1$. Now, 
let $D^{\prime}$ be a $\gamma_{s t}(H-\{v\})$-set. Since $s_{v} \in \mathcal{S}(H-\{v\})$, by Remark 3 we have that $s_{v} \in D^{\prime}$ and no vertex of $H-\{v\}$ is totally protected by $s_{v}$ under $D^{\prime}$, which implies that $D^{\prime} \cup\{v\}$ is a secure total dominating set of $H$ and, as a result, $\gamma_{s t}(H)-1 \leq\left|D^{\prime} \cup\{v\}\right|-1=\left|D^{\prime}\right|=\gamma_{s t}(H-\{v\})$. Therefore, $\gamma_{s t}(H-\{v\})=\gamma_{s t}(H)-1$.

Lemma 3. For any graph $H$ having a universal vertex $v$,

$$
\gamma_{s t}(H)=\gamma(H-\{v\})+1 .
$$

Proof. Let $D$ be a $\gamma(H-\{v\})$-set. Since $v$ is a universal vertex of $H$, it is straightforward that $D \cup\{v\}$ is a secure total dominating set of $H$. Thus, $\gamma_{s t}(H) \leq|D \cup\{v\}|=\gamma(H-\{v\})+1$.

From now on, suppose that $\gamma_{s t}(H) \leq \gamma(H-\{v\})$ and let $S$ be a $\gamma_{s t}(H)$-set. We differentiate the following two cases.

Case 1. $v \in S$. In this case, as $|S| \leq \gamma(H-\{v\})$, we deduce that $S \backslash\{v\}$ is not a dominating set of $H-\{v\}$. Hence, there exists a vertex $y \in V(H-\{v\})$ such that $N(y) \cap S=\{v\}$, which is a contradiction, as $S$ is a 2-dominating set, by Remark 1 .

Case 2. $v \notin S$. In this case, $S$ is a secure total dominating set of $H-\{v\}$ and so $\gamma_{s t}(H-\{v\}) \leq|S| \leq$ $\gamma(H-\{v\})$, which is a contradiction with Remark 2.

Therefore, the result follows.

\section{The Case of Rooted Product Graphs}

Given a graph $G$ of order $\mathrm{n}(G)$ and a graph $H$ with root vertex $v$, the rooted product graph $G \circ_{v} H$ is defined as the graph obtained from $G$ and $H$ by taking one copy of $G$ and $n(G)$ copies of $H$ and identifying the $i^{\text {th }}$ vertex of $G$ with the root vertex $v$ in the $i^{t h}$ copy of $H$ for every $i \in\{1,2, \ldots, \mathrm{n}(G)\}$.

If $H$ or $G$ is a trivial graph, then $G \circ_{v} H$ is equal to $G$ or $H$, respectively. In this sense, hereafter we will only consider graphs $G$ and $H$ of order greater than or equal to two.

For every $x \in V(G), H_{x} \cong H$ will denote the copy of $H$ in $G \circ_{v} H$ containing $x$. The restriction of any set $S \subseteq V\left(G \circ_{v} H\right)$ to $V\left(H_{x}\right)$ will be denoted by $S_{x}$, and the restriction to $V\left(H_{x}-\{x\}\right)$ will be denoted by $S_{x}^{-}$. Hence, $V\left(G \circ_{v} H\right)=\cup_{x \in V(G)} V\left(H_{x}\right)$ and for every $\gamma_{s t}\left(G \circ_{v} H\right)$-set $S$ we have that

$$
\gamma_{s t}\left(G \circ_{v} H\right)=|S|=\sum_{x \in V(G)}\left|S_{x}\right|=\sum_{x \in V(G)}\left|S_{x}^{-}\right|+|S \cap V(G)| .
$$

Theorem 1. For any graphs $G$ and $H$ with no isolated vertex and any $v \in V(H)$,

$$
\gamma_{s t}\left(G \circ \circ_{v} H\right) \leq \mathrm{n}(G) \gamma_{s t}(H)
$$

Furthermore, if $v \notin \mathcal{S}(H)$, then

$$
\gamma_{s t}(G \circ v) \leq \gamma_{s t}(G)+\mathrm{n}(G) \gamma_{s t}(H-\{v\}) .
$$

Proof. Let $D$ be a $\gamma_{s t}(H)$-set and $S \subseteq V\left(G \circ_{v} H\right)$ such that $S_{x}$ is the subset of $V\left(H_{x}\right)$ induced by $D$ for every $x \in V(G)$. Since $S$ is a secure total dominating set of $G \circ_{v} H$, we deduce that $\gamma_{s t}\left(G \circ_{v} H\right) \leq$ $\sum_{x \in V(G)}\left|S_{x}\right|=\mathrm{n}(G) \gamma_{s t}(H)$.

Now, assume that $v \notin \mathcal{S}(H)$. Let $W$ be a $\gamma_{s t}(H-\{v\})$-set and $S^{\prime} \subseteq V\left(G \circ_{v} H\right) \backslash V(G)$ such that $S_{x}^{\prime}$ is the subset of $V\left(H_{x}-\{x\}\right)$ induced by $W$ for every $x \in V(G)$. Since for any $\gamma_{s t}(G)$-set $X$, we have that $X \cup S^{\prime}$ is a secure total dominating set of $G \circ_{v} H$, we deduce that $\gamma_{s t}\left(G \circ \circ_{v} H\right) \leq\left|X \cup S^{\prime}\right|=$ $\gamma_{s t}(G)+\mathbf{n}(G) \gamma_{s t}(H-\{v\})$. 
We now proceed to analyse three cases in which it is not difficult to give closed formulas for $\gamma_{s t}\left(G \circ_{v} H\right)$. Specifically, we consider the cases in which the root vertex $v$ is a support vertex, a strong leaf, or a universal vertex.

Theorem 2. The following statements hold for any graphs $G$ and $H$ with no isolated vertex.

(i) If $v \in \mathcal{S}(H)$, then $\gamma_{s t}\left(G \circ_{v} H\right)=\mathrm{n}(G) \gamma_{s t}(H)$. Furthermore, $\left|D_{x}\right|=\gamma_{s t}(H)$ for every $\gamma_{s t}\left(G \circ_{v} H\right)$-set $D$ and every $x \in V(G)$.

(ii) If $v \in V(H)$ is a universal vertex, then $\gamma_{s t}\left(G \circ_{v} H\right)=\mathrm{n}(G) \gamma_{s t}(H)$.

(iii) If $v \in \mathcal{L}_{s}(H)$, then $\gamma_{s t}(G \circ v)=\gamma(G)+\mathrm{n}(G)\left(\gamma_{s t}(H)-1\right)$.

Proof. Let $D$ be a $\gamma_{s t}\left(G \circ_{v} H\right)$-set. Let us first consider the case where $v \in \mathcal{S}(H)$. Since $x \in \mathcal{S}\left(G \circ_{v} H\right)$ for every $x \in V(G)$, by Remark 3 we deduce that $D_{x}$ is a secure total dominating set of $H_{x}$, and as a consequence $\left|D_{x}\right| \geq \gamma_{s t}\left(H_{x}\right)$ for every $x \in V(G)$. Hence, $\gamma_{s t}\left(G \circ_{v} H\right)=\sum_{x \in V(G)}\left|D_{x}\right| \geq \mathrm{n}(G) \gamma_{s t}(H)$. Now, if $\left|D_{x}\right| \geq \gamma_{s t}\left(H_{x}\right)+1$ for some $x \in V(G)$, then $\gamma_{s t}\left(G \circ_{v} H\right)>\mathrm{n}(G) \gamma_{s t}(H)$, which contradicts Theorem 1. Therefore, (i) follows.

Let us now consider the case where $v \notin \mathcal{S}(H)$ is a universal vertex. Let $x \in V(G)$. If $x \in D_{x}$, then $D_{x}$ is a secure total dominating set of $H_{x}$ and, as a result, $\left|D_{x}\right| \geq \gamma_{s t}\left(H_{x}\right)$. Now, if $x \notin D_{x}$, then $D_{x}^{-}$is a secure total dominating set of $H_{x}-\{x\}$, and so Remark 2 and Lemma 3 lead to $\left|D_{x}\right| \geq$ $\gamma_{s t}\left(H_{x}-\{x\}\right) \geq \gamma\left(H_{x}-\{x\}\right)+1=\gamma_{s t}\left(H_{x}\right)$. Hence, $\gamma_{s t}\left(G \circ_{v} H\right)=\sum_{x \in V(G)}\left|D_{x}\right| \geq \mathrm{n}(G) \gamma_{s t}(H)$ and (ii) follows by Theorem 1.

From now on we assume that $v \in \mathcal{L}_{S}(H)$. Let $s_{x} \in V\left(H_{x}\right)$ be the support of $x$ in $H_{x}$ for every $x \in V(G)$. Since $x \in \mathcal{L}_{\mathcal{S}}\left(H_{x}\right)$, we have that $s_{x} \in \mathcal{S}\left(H_{x}-\{x\}\right) \cap D$. Hence, by Remark 3 we deduce that $D_{x}^{-}$is a secure total dominating set of $H_{x}-\{x\}$, and by Lemma 2 we have that $\left|D_{x}^{-}\right| \geq \gamma_{s t}\left(H_{x}-\{x\}\right)=$ $\gamma_{s t}(H)-1$. Moreover, since $N(x) \cap D_{x}=\left\{s_{x}\right\}$ for every $x \in V(G)$, by Remark 1 it follows that every vertex in $V(G) \backslash D$ has to have a neighbour in $V(G) \cap D$, which implies that $V(G) \cap D$ is a dominating set of $G$. Therefore, $\gamma_{s t}\left(G \circ \circ_{v} H\right)=|D|=|D \cap V(G)|+\left|\cup_{x \in V(G)} D_{x}^{-}\right| \geq \gamma(G)+\mathrm{n}(G)\left(\gamma_{s t}(H)-1\right)$.

It remains to show that $\gamma_{s t}\left(G \circ_{v} H\right) \leq \gamma(G)+\mathrm{n}(G)\left(\gamma_{s t}(H)-1\right)$. To this end, let $X$ be a $\gamma(G)$-set, $Y$ a $\gamma_{s t}(H-\{v\})$-set, and $W \subseteq V\left(G \circ_{v} H\right) \backslash V(G)$ such that $W_{x}$ is the subset of $V\left(H_{x}-\{x\}\right)$ induced by $Y$ for every $x \in V(G)$. Notice that $s_{x} \in W_{x}$. In order to show that $S=X \cup W$ is a secure total dominating set of $G \circ_{v} H$, we only need to observe that every vertex in $V(G) \backslash S$ is totally protected under $S$ by any neighbour in $X$, while every $w \in V\left(H_{x}\right) \backslash W_{x}$ is totally protected under $S$ by some neighbour in $W_{x}$. Thus, $\gamma_{s t}\left(G \circ_{v} H\right) \leq|S|=\gamma(G)+\mathrm{n}(G) \gamma_{s t}(H-\{v\})$, and by Lemma 2 we deduce that $\gamma_{s t}\left(G \circ_{v} H\right) \leq \gamma(G)+\mathrm{n}(G)\left(\gamma_{s t}(H)-1\right)$. Therefore, (iii) follows.

Given two graphs $G$ and $G^{\prime}$, the corona graph $G \odot G^{\prime}$ can be seen as a rooted product graph $G \circ_{v} H$ where $H$ is the join (The join graph $G^{\prime}+G^{\prime \prime}$ is the graph obtained from $G^{\prime}$ and $G^{\prime \prime}$ by joining each vertex of $G^{\prime}$ to all vertices of $G^{\prime \prime}$ ) graph $K_{1}+H$ and $v$ is the vertex of $K_{1}$. Therefore, Lemma 3 and Theorem 2 (ii) lead to the following result on corona graphs.

Theorem 3. If $G$ is a graph with no isolated vertex, then for every nontrivial graph $G^{\prime}$,

$$
\gamma_{s t}\left(G \odot G^{\prime}\right)=\mathrm{n}(G)\left(\gamma\left(G^{\prime}\right)+1\right)
$$

As we will see later, the behaviour of $\gamma_{s t}(G \circ v)$ changes depending on whether the root vertex $v$ is a weak leaf or not. First we proceed to consider the cases where the root vertex is not a weak leaf.

Lemma 4. Let $S$ be a $\gamma_{s t}\left(G \circ_{v} H\right)$-set and $x \in V(G)$. If $v \notin \mathcal{L}_{w}(H)$, then the following statements hold.

- $\left|S_{x}\right| \geq \gamma_{s t}(H)-2$.

- If $\left|S_{x}\right|=\gamma_{s t}(H)-2$, then $N[x] \cap S_{x}=\varnothing$. 
Proof. Let $x \in V(G)$. Notice that every vertex in $V\left(H_{x}\right) \backslash(S \cup\{x\})$ is totally protected under $S$ by some vertex in $S_{x}$. Now, suppose that $\left|S_{x}\right| \leq \gamma_{s t}(H)-3$ and let $y \in N(x) \cap V\left(H_{x}\right)$. If $y \notin S_{x}$, then $S_{x} \cup\{x, y\}$ is a secure total dominating set of $H_{x}$ of cardinality at most $\gamma_{s t}(H)-1$, which is a contradiction. Assume that $N(x) \cap V\left(H_{x}\right) \subseteq S_{x}$. If $N(x) \cap V\left(H_{x}\right)=\{y\}$, then $x \in \mathcal{L}_{s}\left(H_{x}\right)$ and $y \in$ $\mathcal{S}\left(G \circ_{v} H\right)$. Thus, by Remark 3 no vertex in $V\left(H_{x}\right)$ is totally protected by $y$ under $S$, and so $S_{x} \cup\{x\}$ is a secure total dominating set of $H_{x}$ of cardinality at most $\gamma_{s t}(H)-2$, which is a contradiction. Finally, if $\left|N(x) \cap V\left(H_{x}\right)\right| \geq 2$, then $S_{x} \cup\{x\}$ is a secure total dominating set of $H_{x}$ and, as above, we arrive to a contradiction. Therefore, $\left|S_{x}\right| \geq \gamma_{s t}(H)-2$.

Now, assume that $\left|S_{x}\right|=\gamma_{s t}(H)-2$. First, suppose that $x \in S$. Notice that if $N(x) \cap V\left(H_{x}\right) \subseteq S_{x}$, then $S_{x}$ is a secure total dominating set of $H_{x}$, which is a contradiction. Hence, there exists $y \in$ $\left(N(x) \cap V\left(H_{x}\right)\right) \backslash S_{x}$, and so $S_{x} \cup\{y\}$ is a secure total dominating set of $H_{x}$ and $\left|S_{x} \cup\{y\}\right|=\gamma_{s t}(H)-1$, which is a contradiction. Thus, $x \notin S$. Now, suppose that $N(x) \cap S_{x} \neq \varnothing$. If there exists $z \in$ $\left(N(x) \cap V\left(H_{x}\right)\right) \backslash S_{x}$, then $S_{x} \cup\{z\}$ is a secure total dominating set of $H_{x}$ and $\left|S_{x} \cup\{z\}\right|=\gamma_{s t}(H)-1$, which is a contradiction. Now, if $N(x) \cap V\left(H_{x}\right) \subseteq S_{x}$, then one can easily check that $S_{x} \cup\{x\}$ is a secure total dominating set of $H_{x}$, which is a contradiction again, as $\left|S_{x} \cup\{x\}\right|=\gamma_{s t}(H)-1$. Therefore, $N(x) \cap V\left(H_{x}\right) \cap S=\varnothing$.

From Lemma 4 we deduce that if $v \notin \mathcal{L}_{w}(H)$, then any $\gamma_{s t}\left(G \circ_{v} H\right)$-set $S$ induces a partition $\left\{\mathcal{A}_{S}, \mathcal{B}_{S}, \mathcal{C}_{S}\right\}$ of $V(G)$ as follows.

$$
\begin{gathered}
\mathcal{A}_{S}=\left\{x \in V(G):\left|S_{x}\right| \geq \gamma_{s t}(H)\right\}, \\
\mathcal{B}_{S}=\left\{x \in V(G):\left|S_{x}\right|=\gamma_{s t}(H)-1\right\}, \\
\mathcal{C}_{S}=\left\{x \in V(G):\left|S_{x}\right|=\gamma_{s t}(H)-2\right\} .
\end{gathered}
$$

The following corollary is a direct consequence of Theorem 2 (i).

Corollary 1. Let $S$ be a $\gamma_{s t}\left(G \circ_{v} H\right)$-set. If $\mathcal{B}_{S} \cup \mathcal{C}_{S} \neq \varnothing$, then $v \notin \mathcal{S}(H)$.

Lemma 5. Let $S$ be a $\gamma_{s t}\left(G \circ_{v} H\right)$-set, where $v \notin \mathcal{L}_{w}(H)$. If $\mathcal{C}_{S} \neq \varnothing$, then $\gamma_{s t}(H-\{v\})=\gamma_{s t}(H)-2$.

Proof. By Lemma 4, if $x \in \mathcal{C}_{S}$, then $N[x] \cap S_{x}=\varnothing$, which implies that $S_{x}^{-}$is a secure total dominating set of $H_{x}-\{x\}$ of cardinality $\left|S_{x}^{-}\right|=\left|S_{x}\right|=\gamma_{s t}\left(H_{x}\right)-2$. Hence, $x \notin \mathcal{S}\left(H_{x}\right)$ and $\gamma_{s t}\left(H_{x}-\{x\}\right) \leq$ $\left|S_{x}^{-}\right|=\gamma_{s t}\left(H_{x}\right)-2$. Notice that Lemma 2 leads to $x \notin \mathcal{L}_{S}\left(H_{x}\right)$. Thus, by Lemma 1 we conclude that $\gamma_{s t}\left(H_{x}-\{x\}\right)=\gamma_{s t}\left(H_{x}\right)-2$. Therefore, the result follows.

The following result states the intervals in which the secure total domination number of a rooted product graph can be found.

Theorem 4. Let $G$ and $H$ be two graphs with no isolated vertex. At least one of the following statements holds for every $v \in V(H) \backslash \mathcal{L}_{w}(H)$.

(i) $\gamma_{s t}(G \circ v)=\mathrm{n}(G) \gamma_{s t}(H)$.

(ii) $\mathrm{n}(G)\left(\gamma_{s t}(H)-1\right) \leq \gamma_{s t}(G \circ v) \leq \gamma_{s t}(G)+\mathrm{n}(G)\left(\gamma_{s t}(H)-1\right)$.

(iii) $\gamma_{\times 2}(G)+\mathrm{n}(G)\left(\gamma_{s t}(H)-2\right) \leq \gamma_{s t}\left(G \circ_{v} H\right) \leq \gamma_{s t}(G)+\mathrm{n}(G)\left(\gamma_{s t}(H)-2\right)$.

Proof. Let $S$ be a $\gamma_{s t}\left(G \circ_{v} H\right)$-set and consider the partition $\left\{\mathcal{A}_{S}, \mathcal{B}_{S}, \mathcal{C}_{S}\right\}$ of $V(G)$ defined above. We differentiate the following four cases.

Case $1 . \mathcal{B}_{S} \cup \mathcal{C}_{S}=\varnothing$. In this case, for any $x \in V(G)$ we have that $\left|S_{x}\right| \geq \gamma_{s t}(H)$ and, as a consequence, $\gamma_{s t}(G \circ v) \geq \mathrm{n}(G) \gamma_{s t}(H)$. Thus, Theorem 1 leads to (i).

Case 2. $\mathcal{B}_{S} \neq \varnothing$ and $\mathcal{C}_{S}=\varnothing$. In this case, for any $x \in V(G)$ we have that $\left|S_{x}\right| \geq \gamma_{s t}(H)-1$ and, as a result, $\gamma_{s t}\left(G \circ_{v} H\right) \geq \mathrm{n}(G)\left(\gamma_{s t}(H)-1\right)$. 
In order to conclude the proof of (ii), we proceed to show that $\gamma_{s t}\left(G \circ_{v} H\right) \leq \gamma_{s t}(G)+$ $\mathrm{n}(G)\left(\gamma_{s t}(H)-1\right)$. To this end, we fix $x^{\prime} \in \mathcal{B}_{S}, y_{x^{\prime}} \in V\left(H_{x^{\prime}}\right) \cap N\left(x^{\prime}\right)$, a $\gamma_{s t}(G)$-set $D$ and define a subset $W$ of vertices of $G \circ_{v} H$ as follows.

(a) If $x^{\prime} \notin S$, then for any $x \in V(G)$ we set $W \cap V(G)=D$ and $W_{x}^{-}$is induced by $S_{x^{\prime}}^{-}=S_{x^{\prime}}$. It is readily seen that the set $W$ constructed in this manner is a secure total dominating set of $G \circ_{v} H$ and so $\gamma_{s t}\left(G \circ_{v} H\right) \leq|W|=|D|+\mathrm{n}(G)\left|S_{x^{\prime}}\right|=\gamma_{s t}(G)+\mathrm{n}(G)\left(\gamma_{s t}(H)-1\right)$.

(b) Assume that $x^{\prime} \in S$. If $x \in V(G) \backslash \mathcal{L}(G)$, then $W_{x}$ is induced by $S_{x^{\prime}}$, while if $x \in \mathcal{L}(G)$, then $W_{x}$ is induced by $S_{x^{\prime}} \cup\left\{y_{x^{\prime}}\right\}$. It is readily seen that the set $W$ constructed in this manner is a secure total dominating set of $G \circ_{v} H$ and, as a result, $\gamma_{s t}\left(G \circ_{v} H\right) \leq|W|=|\mathcal{L}(G)|+\mathrm{n}(G)\left|S_{x^{\prime}}\right| \leq$ $\gamma_{s t}(G)+\mathrm{n}(G)\left(\gamma_{s t}(H)-1\right)$

Case 3. $\mathcal{B}_{S}=\varnothing$ and $\mathcal{C}_{S} \neq \varnothing$. By Corollary $1, v \notin \mathcal{S}(H)$, and by Lemma 5 we have that $\gamma_{s t}(H-\{v\})=$ $\gamma_{s t}(H)-2$. Hence, by Theorem 1 we conclude that $\gamma_{s t}(G \circ v) \leq \gamma_{s t}(G)+\mathrm{n}(G)\left(\gamma_{s t}(H)-2\right)$.

From Lemma 4 we deduce that $\mathcal{A}_{S}$ is a 2-dominating set of $G$. Hence, $\gamma_{s t}\left(G \circ_{v} H\right) \geq$ $\left|\mathcal{A}_{S}\right| \gamma_{s t}(H)+\left|\mathcal{C}_{S}\right|\left(\gamma_{s t}(H)-2\right)=2\left|\mathcal{A}_{S}\right|+\mathrm{n}(G)\left(\gamma_{s t}(H)-2\right) \geq 2 \gamma_{2}(G)+\mathrm{n}(G)\left(\gamma_{s t}(H)-2\right) \geq$ $\gamma_{\times 2}(G)+\mathrm{n}(G)\left(\gamma_{s t}(H)-2\right)$. Therefore, in this case (iii) holds.

Case 4. $\mathcal{B}_{S} \neq \varnothing$ and $\mathcal{C}_{S} \neq \varnothing$. By Corollary $1, v \notin \mathcal{S}(H)$, and by Lemma $5, \gamma_{s t}(H-\{v\})=\gamma_{s t}(H)-2$. Thus, by Theorem 1 we conclude that $\gamma_{s t}(G \circ v) \leq \gamma_{s t}(G)+\mathrm{n}(G)\left(\gamma_{s t}(H)-2\right)$.

In order to conclude that in this case (iii) holds, let us define a double dominating set $D$ of $G$ such that $|D| \leq 2\left|\mathcal{A}_{S}\right|+\left|\mathcal{B}_{S}\right|$. Set $D$ has minimum cardinality among the sets satisfying that $\mathcal{A}_{S} \cup \mathcal{B}_{S} \subseteq D$ and for any $x \in \mathcal{A}_{S}$, if $N(x) \cap \mathcal{C}_{S} \neq \varnothing$, then there exists $x^{\prime} \in N(x) \cap \mathcal{C}_{S} \cap D$. Notice that every vertex in $\mathcal{A}_{S}$ is dominated by at least one vertex in $D$ and, by Lemma 4 , every vertex in $\mathcal{C}_{S}$ is dominated by at least two vertices in $\mathcal{A}_{S} \cup \mathcal{B}_{S} \subseteq D$. Furthermore, if there exists one vertex $x \in \mathcal{B}_{S}$ such that $N(x) \cap \mathcal{A}_{S} \cap \mathcal{B}_{S}=\varnothing$, then $S_{x}$ is a secure total dominating set of $H_{x}$, which is a contradiction, as $\left|S_{x}\right|=$ $\gamma_{s t}\left(H_{x}\right)-1$. Hence, $D$ is a double dominating set of $G$. Therefore, $\gamma_{s t}\left(G \circ_{v} H\right)=|S| \geq\left|\mathcal{A}_{S}\right| \gamma_{s t}(H)+$ $\left|\mathcal{B}_{S}\right|\left(\gamma_{s t}(H)-1\right)+\left|\mathcal{C}_{S}\right|\left(\gamma_{s t}(H)-2\right) \geq|D|+\mathrm{n}(G)\left(\gamma_{s t}(H)-2\right) \geq \gamma_{\times 2}(G)+\mathrm{n}(G)\left(\gamma_{s t}(H)-2\right)$.

The bounds given in the previous theorem are tight. To see this, we consider the following examples where $H_{1}$ and $H_{2}$ are the graphs shown in Figure 3.

- $\gamma_{s t}\left(G \circ_{v} P_{7}\right)=\mathrm{n}(G)\left(\gamma_{s t}\left(P_{7}\right)-1\right)$, where $v$ is the central vertex of $P_{7}$ and $G$ is a graph with $\delta(G) \geq 2$.

- $\gamma_{s t}\left(K_{r} \circ_{v} H_{1}\right)=2+r(3-1)=\gamma_{s t}\left(K_{r}\right)+n\left(K_{r}\right)\left(\gamma_{s t}\left(H_{1}\right)-1\right)$, where $r \geq 2$.

- Theorem 5 gives some conditions to achieve the equalities $\gamma_{s t}\left(G \circ_{v} H\right)=\gamma_{s t}(G)+\mathrm{n}(G)\left(\gamma_{s t}(H)-\right.$ $2)=\gamma_{\times 2}(G)+\mathrm{n}(G)\left(\gamma_{s t}(H)-2\right)$. In this case we can take $H \cong H_{2}$.

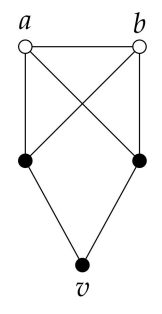

$H_{1}$

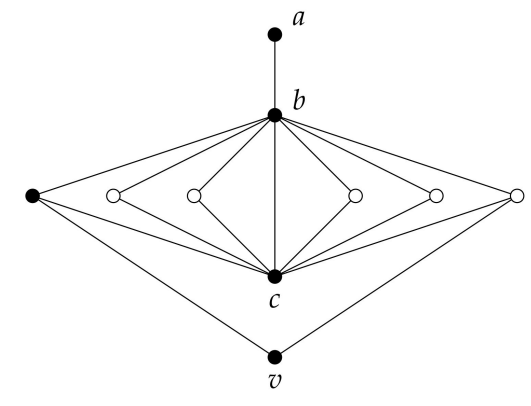

$\mathrm{H}_{2}$

Figure 3. The set of black-coloured vertices forms a $\gamma_{s t}\left(H_{i}\right)$-set for $i \in\{1,2\}$. The set $\{a, b\}$ is a $\gamma_{s t}\left(H_{1}-\{v\}\right)$-set, while $\{a, b, c\}$ is a $\gamma_{s t}\left(H_{2}-\{v\}\right)$-set.

We now consider some particular cases in which we impose some additional restrictions on $G$ and $H$. We begin with an immediate consequence of Theorem 4. 
Theorem 5. Let $G$ and $H$ be two graphs with no isolated vertex and $v \in V(H) \backslash \mathcal{L}_{w}(H)$. If $\gamma_{s t}(H-\{v\})=$ $\gamma_{s t}(H)-2$ and $\gamma_{s t}(G)=\gamma_{\times 2}(G)$, then

$$
\gamma_{s t}\left(G \circ_{v} H\right)=\gamma_{s t}(G)+\mathrm{n}(G)\left(\gamma_{s t}(H)-2\right)
$$

Proof. If $\gamma_{s t}(H-\{v\})=\gamma_{s t}(H)-2$, then $v \notin \mathcal{S}(H)$ and Theorem 1 leads to $\gamma_{s t}\left(G \circ_{v} H\right) \leq \gamma_{s t}(G)+$ $\mathrm{n}(G)\left(\gamma_{s t}(H)-2\right)$. Thus, by Theorem 4 we conclude that if $\gamma_{s t}(G)=\gamma_{\times 2}(G)$, then $\gamma_{s t}\left(G \circ_{v} H\right)=$ $\gamma_{s t}(G)+\mathrm{n}(G)\left(\gamma_{s t}(H)-2\right)$.

The following result considers the case in which $\gamma_{s t}(H-\{v\}) \geq \gamma_{s t}(H)-1$.

Theorem 6. Let $G$ and $H$ be two graphs with no isolated vertex and $v \in V(H) \backslash \mathcal{L}_{w}(H)$. If $\gamma_{s t}(H-\{v\}) \geq$ $\gamma_{s t}(H)-1$, then

$$
\mathrm{n}(G)\left(\gamma_{s t}(H)-1\right) \leq \gamma_{s t}\left(G \circ \circ_{v} H\right) \leq \mathrm{n}(G) \gamma_{s t}(H)
$$

Now, if $\delta(G) \geq 2$ and $\gamma_{s t}(H-\{v\}) \geq \gamma_{s t}(H)$, then $\gamma_{s t}\left(G \circ_{v} H\right)=\mathrm{n}(G)\left(\gamma_{s t}(H)-1\right)$ or $\gamma_{s t}\left(G \circ_{v} H\right)=$ $\mathrm{n}(G) \gamma_{s t}(H)$.

Proof. Let $S$ be a $\gamma_{s t}\left(G \circ_{v} H\right)$-set and assume that $\gamma_{s t}(H-\{v\}) \geq \gamma_{s t}(H)-1$. By Lemma 5 we have that $\mathcal{C}_{S}=\varnothing$, and so Lemma 4 leads to $\left|S_{x}\right| \geq \gamma_{s t}\left(H_{x}\right)-1$ for every $x \in V(G)$. Thus, $\gamma_{s t}\left(G \circ_{v} H\right)=$ $\sum_{x \in V(G)}\left|S_{x}\right| \geq \mathrm{n}(G)\left(\gamma_{s t}(H)-1\right)$. Therefore, Theorem 1 leads to $\mathrm{n}(G)\left(\gamma_{s t}(H)-1\right) \leq \gamma_{s t}\left(G \circ_{v} H\right) \leq$ $\mathrm{n}(G) \gamma_{s t}(H)$.

From now on we assume that $\delta(G) \geq 2$ and $\gamma_{s t}(H-\{v\}) \geq \gamma_{s t}(H)$. Let us distinguish between two cases, according to whether or not $\gamma_{s t}(H-\{v\})>\gamma_{s t}(H)$.

Case 1. $\gamma_{s t}(H-\{v\})>\gamma_{s t}(H)$. We define a set $D \subseteq V\left(G \circ_{v} H\right)$ as follows. For any $x \in V(G) \backslash S$ we take $D \cap V\left(H_{x}\right)$ as a $\gamma_{s t}\left(H_{x}\right)$-set, while for any $x \in V(G) \cap S$ we set $D \cap V\left(H_{x}\right)=S_{x}$. Notice that $D$ is a secure total dominating set of $G \circ_{v} H$. Now, if there exists a vertex $x \in V(G) \backslash S$, then the set $S_{x}^{-}$ is a secure total dominating set of $H_{x}-\{x\}$. Hence, $\left|S_{x}\right|=\left|S_{x}^{-}\right| \geq \gamma_{s t}(H-\{x\})>\gamma_{s t}\left(H_{x}\right)=\left|D_{x}\right|$, and so $|D|<|S|$, which is a contradiction. Thus, $V(G) \subseteq S$.

If $\left|S_{x}\right| \geq \gamma_{s t}(H)$ for every $x \in V(G)$, then Theorem 1 leads to $\gamma_{s t}\left(G \circ_{v} H\right)=\mathrm{n}(G) \gamma_{s t}(H)$. Suppose that there exists a vertex $x \in V(G)$ such that $\left|S_{x}\right| \leq \gamma_{s t}(H)-1$. We define a set $D^{\prime} \subseteq$ $V\left(G \circ_{v} H\right)$ as follows. For every $z \in V(G)$, the restriction of $D^{\prime}$ to $V\left(H_{z}\right)$ is induced by $S_{x}$. Notice that $V(G) \subseteq D^{\prime}$ and, if $\delta(G) \geq 2$, then every vertex in $V\left(H_{z}\right) \backslash D^{\prime}$ is totally protected under $D^{\prime}$ by some vertex in $D_{z}^{\prime}$, which implies that $D^{\prime}$ is a secure total dominating set of $G \circ_{v} H$. Therefore, $\gamma_{s t}(G \circ v) \leq\left|D^{\prime}\right| \leq \mathrm{n}(G)\left(\gamma_{s t}(H)-1\right)$, concluding that $\gamma_{s t}(G \circ v H)=\mathrm{n}(G)\left(\gamma_{s t}(H)-1\right)$.

Case 2. $\gamma_{s t}(H-\{v\})=\gamma_{s t}(H)$. First, assume that $V(G) \cap S=\varnothing$. Since $S_{x}^{-}$is a secure total dominating set of $H_{x}-\{x\}$ for every $x \in V(G)$, we have that $\gamma_{s t}\left(G \circ_{v} H\right)=\sum_{x \in V(G)}\left|S_{x}^{-}\right| \geq \sum_{x \in V(G)} \gamma_{s t}(H-$ $\{x\})=\mathrm{n}(G) \gamma_{s t}(H-\{v\})=\mathrm{n}(G) \gamma_{s t}(H)$, and so Theorem 1 leads to $\gamma_{s t}\left(G \circ_{v} H\right)=\mathrm{n}(G) \gamma_{s t}(H)$.

Now, assume that there exists $y \in V(G) \cap S$. Notice that Lemma 5 leads to $\mathcal{C}_{S}=\varnothing$. Hence, $y \in \mathcal{A}_{S} \cup \mathcal{B}_{S}$. If $y \in \mathcal{B}_{S}$, we define a set $D^{\prime} \subseteq V\left(G \circ_{v} H\right)$ as follows. For every $z \in V(G)$, the restriction of $D^{\prime}$ to $V\left(H_{z}\right)$ is induced by $S_{y}$. As in Case 1 , we deduce that $D^{\prime}$ is a secure total dominating set of $G \circ_{v} H$ and so we can conclude that $\gamma_{s t}\left(G \circ_{v} H\right)=\left|D^{\prime}\right|=\mathrm{n}(G)\left(\gamma_{s t}(H)-1\right)$. Finally, if $\mathcal{B}_{S}=\varnothing$, then $V(G)=\mathcal{A}_{S}$ and by Theorem 1 we conclude that $\gamma_{s t}(G \circ v)=|S|=\mathrm{n}(G) \gamma_{s t}(H)$.

Now, we consider a particular case in which $\gamma_{s t}(H-\{v\})=\gamma_{s t}(H)$.

Theorem 7. Let $G$ be a graph with no isolated vertex. Let $H$ be a graph and $v \in V(H)$ such that $\gamma_{s t}(H-$ $\{v\})=\gamma_{s t}(H)$. If $v \notin S$ for every $\gamma_{s t}(H)$-set $S$, then

$$
\gamma_{s t}(G \circ v)=\mathrm{n}(G) \gamma_{s t}(H)
$$


Proof. Assume that $v \notin S$ for every $\gamma_{s t}(H)$-set $S$. Notice that $v \notin \mathcal{L}(H) \cup \mathcal{S}(H)$. Let $D$ be a $\gamma_{s t}\left(G \circ_{v} H\right)$-set. By Lemma 5 we conclude that $\mathcal{C}_{D}=\varnothing$. Now, if $\mathcal{B}_{D}=\varnothing$, then by analogy to Case 1 in the proof of Theorem 4 it follows that $\gamma_{s t}\left(G \circ_{v} H\right)=\mathrm{n}(G) \gamma_{s t}(H)$.

Suppose that there exists a vertex $x \in \mathcal{B}_{D}$. If $x \notin D$, then $D_{x}^{-}$is a secure total dominating set of $H_{x}-\{x\}$, which implies that $\gamma_{s t}(H-\{v\})=\gamma_{s t}\left(H_{x}-\{x\}\right) \leq\left|D_{x}^{-}\right|=\left|D_{x}\right|=\gamma_{s t}(H)-1$, which is a contradiction. Hence, $x \in D$. Now, if $N(x) \cap V\left(H_{x}\right) \subseteq D$, then $D_{x}$ is a secure total dominating set of $H_{x}$ and so $\gamma_{s t}\left(H_{x}\right) \leq\left|D_{x}\right|=\gamma_{s t}\left(H_{x}\right)-1$, which is a contradiction. Finally, if there exists $x^{\prime} \in N(x) \cap V\left(H_{x}\right) \backslash D$, then $D_{x}^{\prime}=D_{x} \cup\left\{x^{\prime}\right\}$ is a secure total dominating set of $H_{x}$ of cardinality $\gamma_{s t}\left(H_{x}\right)$ and $x \in D_{x}^{\prime}$, which is a contradiction again. Therefore, $\mathcal{B}_{D}=\varnothing$, and we are done.

The Case in Which the Root Vertex Is a Weak Leaf

The first part of this section is devoted to the case in which the support vertex of the root $v$ has degree greater than or equal to three. From Remark 4 we learned that if $v \in \mathcal{L}_{w}(H), N(v)=\{s\}$ and $|N(s)| \geq 3$, then the gap $\gamma_{s t}(H)-\gamma_{s t}(H-\{v\})$ could be arbitrarily large.

Remark 5. Let $H$ be a graph with no isolated vertex, $v \in \mathcal{L}_{w}(H)$ and $N(v)=\{s\}$. If $|N(s)| \geq 3$, then

$$
\gamma_{s t}(H) \geq \gamma_{s t}(H-\{v\})
$$

Proof. Let $S$ be a $\gamma_{s t}(H)$-set. By Remark 3, we have that $v, s \in S$. If $N(s) \subseteq S$, then since $|N(s)| \geq 3$, we deduce $S \backslash\{v\}$ is a secure total dominating set of $H-\{v\}$. Hence, $\gamma_{s t}(H-\{v\}) \leq|S \backslash\{v\}|<$ $\gamma_{s t}(H)$. Now, if there exists $u \in N(s) \backslash S$, then $(S \backslash\{v\}) \cup\{u\}$ is also a secure total dominating set of $H-\{v\}$. Thus, $\gamma_{s t}(H-\{v\}) \leq|(S \backslash\{v\}) \cup\{u\}|=\gamma_{s t}(H)$. Therefore, the result follows.

By Remarks 4 and 5, it seems reasonable to express $\gamma_{s t}\left(G \circ_{v} H\right)$ in terms of $\gamma_{s t}(H-\{v\})$ rather than $\gamma_{s t}(H)$. To this end, we consider the following lemma.

Lemma 6. Let $S$ be a $\gamma_{s t}\left(G \circ_{v} H\right)$-set. If $v \in \mathcal{L}_{w}(H), N(v)=\{s\}$ and $|N(s)| \geq 3$, then $\left|S_{x}\right| \geq \gamma_{s t}(H-$ $\{v\}$ ) for every $x \in V(G)$

Proof. Let $x \in V(G)$. Notice that every vertex in $V\left(H_{x}\right) \backslash(S \cup\{x\})$ is totally protected under $S$ by some vertex in $S_{x}$. Now, suppose that $\left|S_{x}\right|<\gamma_{s t}(H-\{v\})$ and let $N(x) \cap V\left(H_{x}\right)=\left\{s_{x}\right\}$. If $x \notin S$, then $S_{x}^{-}$is a secure total dominating set of $H_{x}-\{x\}$, which is a contradiction as $\left|S_{x}^{-}\right|=\left|S_{x}\right|<$ $\gamma_{s t}(H-\{v\})=\gamma_{s t}\left(H_{x}-\{x\}\right)$. Hence, $x \in S$. Now, if $N\left(s_{x}\right) \subseteq S$, then we set $S^{\prime}=\left(S_{x} \backslash\{x\}\right) \cup\left\{s_{x}\right\}$ and otherwise we set $S^{\prime}=\left(S_{x} \backslash\{x\}\right) \cup\{w\}$ for any $w \in N\left(s_{x}\right) \backslash S$. In both cases, $S^{\prime}$ is a secure total dominating set of $H_{x}-\{x\}$ and $\gamma_{s t}\left(H_{x}-\{x\}\right)-1>\left|S_{x}\right|-1 \geq\left|S^{\prime}\right| \geq \gamma_{s t}\left(H_{x}-\{x\}\right)$, which is a contradiction. Therefore, $\left|S_{x}\right| \geq \gamma_{s t}(H-\{v\})$.

By Theorem 1 and Lemma 6, we deduce the next result.

Theorem 8. Let $G$ and $H$ be two graphs with no isolated vertex. If $v \in \mathcal{L}_{w}(H), N(v)=\{s\}$ and $|N(s)| \geq 3$, then

$$
\mathrm{n}(G) \gamma_{s t}(H-\{v\}) \leq \gamma_{s t}(G \circ v H) \leq \min \left\{\mathrm{n}(G) \gamma_{s t}(H), \gamma_{s t}(G)+\mathrm{n}(G) \gamma_{s t}(H-\{v\})\right\}
$$

The following result is an immediate consequence of the theorem above.

Corollary 2. Let $G$ and $H$ be two graphs with no isolated vertex. Let $v \in \mathcal{L}_{w}(H)$ and $N(v)=\{s\}$. If $|N(s)| \geq 3$ and $\gamma_{s t}(H-\{v\})=\gamma_{s t}(H)$, then

$$
\gamma_{s t}(G \circ v)=\mathrm{n}(G) \gamma_{s t}(H)
$$


Theorem 9. Let $G$ be a graph with $\delta(G) \geq 2$ and $H$ a graph with no isolated vertex. Let $v \in \mathcal{L}_{w}(H)$ and $N(v)=\{s\}$. If $|N(s)| \geq 3$ and $N(s) \cap \mathcal{S}(H) \neq \varnothing$, then the following statements hold.

(i) If $s \notin D$ for every $\gamma_{s t}(H-\{v\})$-set $D$, then

$$
\gamma_{s t}\left(G \circ_{v} H\right)=\gamma_{s t}(G)+\mathrm{n}(G) \gamma_{s t}(H-\{v\}) .
$$

(ii) If there exists a $\gamma_{s t}(H-\{v\})$-set $D$ such that $s \in D$, then

$$
\gamma_{s t}(G \circ v) \in\left\{\mathrm{n}(G) \gamma_{s t}\left(H-\{v\}, \gamma(G)+\mathrm{n}(G) \gamma_{s t}(H-\{v\}), \gamma_{t}(G)+\mathrm{n}(G) \gamma_{s t}(H-\{v\})\right\} .\right.
$$

Proof. Let $S$ be a $\gamma_{s t}\left(G \circ_{v} H\right)$-set such that $|S \cap N[V(G)]|$ is maximum. For any vertex $x \in V(G)$, let $\left\{s_{x}\right\}=N(x) \cap V\left(H_{x}\right)$. Let $\left\{\mathcal{M}_{0}, \mathcal{M}_{1}, \mathcal{N}_{0}, \mathcal{N}_{1}\right\}$ be the partition of $V(G)$ defined as follows.

$$
\begin{array}{ll}
\mathcal{M}_{0}=\left\{x \in V(G) \backslash S: s_{x} \in S\right\}, & \mathcal{M}_{1}=\left\{x \in V(G) \cap S: s_{x} \in S\right\}, \\
\mathcal{N}_{0}=\left\{x \in V(G) \backslash S: s_{x} \notin S\right\}, & \mathcal{N}_{1}=\left\{x \in V(G) \cap S: s_{x} \notin S\right\} .
\end{array}
$$

By Theorem 1 we have that $\gamma_{s t}(G \circ v) \leq \gamma_{s t}(G)+\mathrm{n}(G) \gamma_{s t}(H-\{v\})$. Hence, in order to prove (i) we proceed to show that $\gamma_{s t}(G \circ v) \geq \gamma_{s t}(G)+\mathrm{n}(G) \gamma_{s t}(H-\{v\})$. To this end, we need to estimate the gap $\left|S_{x}\right|-\gamma_{s t}(H-\{v\})$. Obviously, if $x \in \mathcal{N}_{0}$, then $\left|S_{x}\right|=\gamma_{s t}(H-\{v\})$. Now, since $N\left(s_{x}\right) \cap$ $\mathcal{S}\left(H_{x}\right) \neq \varnothing$, if $x \in \mathcal{M}_{0} \cup \mathcal{M}_{1}$, then $S_{x}^{-}$is a secure total dominating set of $H_{x}-\{x\}$, and so $\left|S_{x}^{-}\right| \geq$ $\gamma_{s t}\left(H_{x}-\{x\}\right)=\gamma_{s t}(H-\{v\})$. By hypothesis of (i) we deduce that, if $x \in \mathcal{M}_{0}$, then $\left|S_{x}\right| \geq\left|S_{x}^{-}\right|>$ $\gamma_{s t}(H-\{v\})$, while if $x \in \mathcal{M}_{1}$, then $\left|S_{x}\right|>\left|S_{x}^{-}\right|>\gamma_{s t}(H-\{v\})$. We now consider the case $x \in$ $\mathcal{N}_{1}$. By Lemma 6 we have that $\left|S_{x}\right| \geq \gamma_{s t}(H-\{v\})$. If $\left|S_{x}\right|=\gamma_{s t}(H-\{v\})$, then $S_{x}^{-} \cup\left\{s_{x}\right\}$ is a secure total dominating set of $H_{x}-\{x\}$ and $\left|S_{x}^{-} \cup\left\{s_{x}\right\}\right|=\left|S_{x}\right|=\gamma_{s t}(H-\{v\})=\gamma_{s t}\left(H_{x}-\{x\}\right)$, which contradicts the hypothesis of (i). Hence, $x \in \mathcal{N}_{1}$ leads to $\left|S_{x}\right|>\gamma_{s t}(H-\{v\})$.

In summary, we can conclude that if $x \in \mathcal{N}_{0}$, then $\left|S_{x}\right|=\gamma_{s t}(H-\{v\})$, if $x \in \mathcal{M}_{0} \cup \mathcal{N}_{1}$, then $\left|S_{x}\right| \geq \gamma_{s t}(H-\{v\})+1$, while if $x \in \mathcal{M}_{1}$, then $\left|S_{x}\right| \geq \gamma_{s t}(H-\{v\})+2$. We claim that there exists a secure total dominating set $Z$ of $G$ such that $|Z| \leq\left|\mathcal{N}_{1}\right|+\left|\mathcal{M}_{0}\right|+2\left|\mathcal{M}_{1}\right|$.

We define $Z$ as a set of minimum cardinality satisfying that $\mathcal{N}_{1} \cup \mathcal{M}_{0} \cup \mathcal{M}_{1} \subseteq Z$ and for any $x \in \mathcal{M}_{1}$ with $N(x) \cap \mathcal{N}_{0} \neq \varnothing$ there exists $w_{x} \in N(x) \cap \mathcal{N}_{0} \cap Z$. Notice that, by definition, $Z$ is a double dominating set of $G$ and, since $\delta(G) \geq 2$, every vertex in $\mathcal{M}_{1}$ has at least two neighbours in $Z \backslash \mathcal{N}_{0}$ or one neighbour in $Z \cap \mathcal{N}_{0}$. Let $x \in V(G) \backslash Z$. Since $x \in \mathcal{N}_{0}$, there exists $y \in S \cap V(G)=\mathcal{M}_{1} \cap \mathcal{N}_{1} \subseteq Z$ such that $x$ is totally protected under $S$ by $y$. We claim that $Z^{\prime}=(Z \backslash\{y\}) \cup\{x\}$ is a total dominating set of $G$. Since $Z$ is a total dominating set of $G$, we have that every vertex in $V(G) \backslash N(y)$ is dominated by some vertex in $Z^{\prime}$. Now, if there exists $u \in N(y) \cap V(G)$ such that $N(u) \cap S \cap V(G)=\{y\}$, then $u \in \mathcal{M}_{1}$, and so $N(u) \cap Z \cap \mathcal{N}_{0} \neq \varnothing$, concluding that $Z^{\prime}$ is a total dominating set of $G$. Hence, $Z$ is a secure total dominating set of $G$, and as a consequence,

$$
\begin{aligned}
\gamma_{s t}\left(G \circ_{v} H\right) & =\sum_{x \in V(G)}\left|S_{x}\right| \\
& =\sum_{x \in \mathcal{M}_{1}}\left|S_{x}\right|+\sum_{x \in \mathcal{M}_{0}}\left|S_{x}\right|+\sum_{x \in \mathcal{N}_{1}}\left|S_{x}\right|+\sum_{x \in \mathcal{N}_{0}}\left|S_{x}\right| \\
& \geq \sum_{x \in \mathcal{M}_{1}}\left(\gamma_{s t}(H-\{v\})+2\right)+\sum_{x \in \mathcal{M}_{0} \cup \mathcal{N}_{1}}\left(\gamma_{s t}(H-\{v\})+1\right)+\sum_{x \in \mathcal{N}_{0}} \gamma_{s t}(H-\{v\}) \\
& =\sum_{x \in V(G)} \gamma_{s t}(H-\{v\})+\left(2\left|\mathcal{M}_{1}\right|+\left|\mathcal{M}_{0}\right|+\left|\mathcal{N}_{1}\right|\right) \\
& \geq \sum_{x \in V(G)} \gamma_{s t}(H-\{v\})+|Z| \\
& \geq \mathrm{n}(G) \gamma_{s t}(H-\{v\})+\gamma_{s t}(G)
\end{aligned}
$$

Therefore, proof of (i) is complete.

We now proceed to prove (ii). From Lemma 6 we can consider the partition $\left\{R_{0}, R_{1}\right\}$ of $V(G)$ defined as follows.

$$
R_{0}=\left\{x \in V(G):\left|S_{x}\right|=\gamma_{s t}(H-\{v\})\right\}, \quad R_{1}=\left\{x \in V(G):\left|S_{x}\right|>\gamma_{s t}(H-\{v\})\right\}
$$


By assumptions, there exists a $\gamma_{s t}(H-\{v\})$-set $D$ such that $s \in D$. Let $W \subseteq V(G \circ v H) \backslash V(G)$ such that $W_{x}$ is induced by $D$ for every vertex $x \in V(G)$.

If $x \in \mathcal{N}_{0}$, then $S^{\prime}=\left(S \backslash S_{x}\right) \cup W_{x}$ is a $\gamma_{s t}\left(G \circ_{v} H\right)$-set with $\left|S^{\prime} \cap N[V(G)]\right|>|S \cap N[V(G)]|$, which is a contradiction. Hence, $\mathcal{N}_{0}=\varnothing$. If $x \in R_{1} \cap \mathcal{N}_{1}$, then $S^{\prime}=\left(S \backslash S_{x}\right) \cup\left(W_{x} \cup\{x\}\right)$ is a $\gamma_{s t}\left(G \circ o_{v} H\right)$-set with $\left|S^{\prime} \cap N[V(G)]\right|>|S \cap N[V(G)]|$, which is a contradiction. Hence, $R_{1} \cap \mathcal{N}_{1}=\varnothing$, and so $\mathcal{N}_{1} \subseteq R_{0}$. Now, by hypothesis of (ii), $\mathcal{M}_{0} \subseteq R_{0}$. Moreover, if $x \in \mathcal{M}_{1}$, then $S_{x}^{-}$is a secure total dominating set of $H_{x}-\{x\}$, and so $x \in R_{1}$. Therefore, $R_{1}=\mathcal{M}_{1}$ and $R_{0}=\mathcal{M}_{0} \cup \mathcal{N}_{1}$.

Now, we suppose that there exists a vertex $x^{\prime} \in \mathcal{N}_{1}$. Let $W^{\prime} \subseteq V\left(G \circ \circ_{v} H\right)$ such that $W_{x}^{\prime}$ is induced by $S_{x^{\prime}}$ for every vertex $x \in V(G)$. Since $\delta(G) \geq 2$ we have that $W^{\prime}$ is a secure total dominating set of $G \circ_{v} H$ of cardinality $\mathrm{n}(G) \gamma_{s t}(H-\{v\})$. Therefore, $\gamma_{s t}(G \circ v) \leq \mathrm{n}(G) \gamma_{s t}(H-\{v\})$ and by Theorem 8, we deduce that $\gamma_{s t}\left(G \circ_{v} H\right)=\mathrm{n}(G) \gamma_{s t}(H-\{v\})$.

From now on, we assume that $\mathcal{N}_{1}=\varnothing$. Hence, $R_{1}=\mathcal{M}_{1}$ and $R_{0}=\mathcal{M}_{0}$. Let $x \in \mathcal{M}_{1}$. As $N\left(s_{x}\right) \cap \mathcal{S}\left(H_{x}\right) \neq \varnothing$, we have that $S_{x}^{-}$is a secure total dominating set of $H_{x}-\{x\}$, and by hypothesis of (ii) we deduce that $\left|S_{x}^{-}\right|=\gamma_{s t}(H-\{v\})$, which implies that $\left|S_{x}\right|=\gamma_{s t}(H-\{v\})+1$. Hence, $\gamma_{s t}\left(G \circ_{v} H\right)=\left|\mathcal{M}_{1}\right|+\mathrm{n}(G) \gamma_{s t}(H-\{v\})$.

Since $V(G)=\mathcal{M}_{0} \cup \mathcal{M}_{1}$ and $\mathcal{M}_{0} \cap \mathcal{M}_{1}=\varnothing$, by Remark 1 , any vertex in $\mathcal{M}_{0}$ is dominated by at least one vertex in $\mathcal{M}_{1}$. Hence, $\mathcal{M}_{1}$ is a dominating set of $G$ and we differentiate the following two cases.

Case 1 . There exists a $\gamma_{s t}(H-\{v\})$-set $D$ containing $s$, such that no vertex in $N(s) \backslash D$ is necessarily totally protected by $s$ under $D$. Let $W^{\prime \prime} \subseteq V(G \circ v H) \backslash V(G)$ such that $W_{x}^{\prime \prime}$ is induced by $D$ for every vertex $x \in V(G)$. In this case, for every $\gamma(G)$-set $X$ we have that $X \cup W^{\prime \prime}$ is a secure total dominating set of $G \circ_{v} H$. Hence $\left|\mathcal{M}_{1}\right|=\gamma(G)$, and as a consequence, $\gamma_{s t}(G \circ v H)=\gamma(G)+\mathrm{n}(G) \gamma_{s t}(H-\{v\})$.

Case 2. For every $\gamma_{s t}(H-\{v\})$-set $D$ containing $s$, there exists a vertex in $V(H) \backslash D$ that is totally protected uniquely by $s$ under $D$. In this case, any vertex in $\mathcal{M}_{1}$ is dominated by another vertex in $\mathcal{M}_{1}$, which implies that $\mathcal{M}_{1}$ is a total dominating set of $G$. As in Case 1 , let $W^{\prime \prime} \subseteq V(G \circ v) \backslash V(G)$ such that $W_{x}^{\prime \prime}$ is induced by $D$ for every vertex $x \in V(G)$. In this case, for every $\gamma_{t}(G)$-set $X$ we have that $X \cup W^{\prime \prime}$ is a secure total dominating set of $G \circ_{v} H$. Hence $\left|\mathcal{M}_{1}\right|=\gamma_{t}(G)$. Therefore, $\gamma_{s t}(G \circ v)=\gamma_{t}(G)+\mathrm{n}(G) \gamma_{s t}(H-\{v\})$.

From now on we consider the case in which the support vertex of the root $v$ has degree two.

Lemma 7. Let $H$ be a graph with no isolated vertex. If $v \in \mathcal{L}_{w}(H), N(v)=\{s\}$ and $|N(s)|=2$, then $\gamma_{s t}(H-\{v\}) \geq \gamma_{s t}(H)-1$.

Proof. Suppose that $\gamma_{s t}(H-\{v\}) \leq \gamma_{s t}(H)-2$ and let $D$ be a $\gamma_{s t}(H-\{v\})$-set. Since both $s$ and its support vertex in $H-\{v\}$ are included in $D$, we have that $D \cup\{v\}$ is a secure total dominating set of $H$. Hence, $\gamma_{s t}(H) \leq|D \cup\{v\}|=\gamma_{s t}(H-\{v\})+1 \leq \gamma_{s t}(H)-1$, which is a contradiction. Therefore, $\gamma_{s t}(H-\{v\}) \geq \gamma_{s t}(H)-1$, which completes the proof.

Theorem 10. Let $S$ be a $\gamma_{s t}(G \circ v)$-set. If $v \in \mathcal{L}_{w}(H), N(v)=\{s\}$ and $|N(s)|=2$, then for any $x \in V(G)$,

$$
\gamma_{s t}(H)-1 \leq\left|S_{x}\right| \leq \gamma_{s t}(H) .
$$

Therefore, with the assumptions above,

$$
\mathrm{n}(G)\left(\gamma_{s t}(H)-1\right) \leq \gamma_{s t}(G \circ v) \leq \mathrm{n}(G) \gamma_{s t}(H) .
$$

Proof. We first consider the case in which $S_{x}$ is a secure total dominating set of $H_{x}$. Since $x \in \mathcal{L}\left(H_{x}\right)$ we have that $x$ belongs to every $\gamma_{s t}\left(H_{x}\right)$-set. So, $\left|S_{x}\right|=\gamma_{s t}\left(H_{x}\right)=\gamma_{s t}(H)$.

Now, assume that $S_{x}$ is not a secure total dominating set of $H_{x}$. Notice that every vertex in $V\left(H_{x}\right) \backslash(S \cup\{x\})$ is totally protected under $S$ by some vertex in $S_{x}$. Since $\left\{x, s_{x}\right\} \cap S_{x} \neq \varnothing$, we have that $S_{x} \cup\left\{x, s_{x}\right\}$ is a secure total dominating set of $H_{x}$. Hence, $\gamma_{s t}(H)-1=\gamma_{s t}\left(H_{x}\right)-1 \leq \mid S_{x} \cup$ 
$\left\{x, s_{x}\right\}|-1 \leq| S_{x} \mid$. Now, if there exists $x^{\prime} \in V(G)$ such that $\left|S_{x^{\prime}}\right|>\gamma_{s t}(H)$, then for any $\gamma_{s t}\left(H_{x^{\prime}}\right)$-set $D$, we have that $S^{\prime}=\left(S \backslash S_{x^{\prime}}\right) \cup D$ is a secure total dominating set of $G \circ_{v} H$ and $\left|S^{\prime}\right|<|S|$, which is a contradiction. Therefore, $\gamma_{s t}(H)-1 \leq\left|S_{x}\right| \leq \gamma_{s t}(H)$ for every $x \in V(G)$, and since $\gamma_{s t}\left(G \circ \circ_{v} H\right)=$ $\sum_{x \in V(G)}\left|S_{x}\right|$, the result follows.

We now consider the particular case where $\delta(G) \geq 2$. By Lemma 7 we only need to consider two cases according to whether $\gamma_{s t}(H-\{v\}) \geq \gamma_{s t}(H)$ or $\gamma_{s t}(H-\{v\})=\gamma_{s t}(H)-1$. These two cases are discussed in Theorems 11 and 12, respectively.

Theorem 11. Let $G$ be a graph with $\delta(G) \geq 2$ and $H$ a graph with no isolated vertex. Let $v \in \mathcal{L}_{w}(H)$, $N(v)=\{s\}$ and $|N(s)|=2$. If $\gamma_{s t}(H-\{v\}) \geq \gamma_{s t}(H)$, then

$$
\gamma_{s t}\left(G \circ_{v} H\right) \in\left\{\mathrm{n}(G) \gamma_{s t}(H), \mathrm{n}(G)\left(\gamma_{s t}(H)-1\right)\right\} .
$$

Proof. Let $S$ be a $\gamma_{s t}\left(G \circ_{v} H\right)$-set such that $|S|<\mathrm{n}(G) \gamma_{s t}(H)$. For any vertex $x \in V(G)$, let $\left\{s_{x}\right\}=$ $N(x) \cap V\left(H_{x}\right)$ and $\left\{s_{x}^{\prime}\right\}=N\left(s_{x}\right) \backslash\{x\}$. By Theorem 10 there exists a vertex $y \in V(G)$ such that $\left|S_{y}\right|=\gamma_{s t}(H)-1$. If $y \notin S_{y}$, then $S_{y}^{-}$is a secure total dominating set of $H_{y}-\{y\}$ and so $\left|S_{y}^{-}\right|=\left|S_{y}\right|=$ $\gamma_{s t}(H)-1<\gamma_{s t}(H-\{v\})=\gamma_{s t}\left(H_{y}-\{y\}\right)$, which is a contradiction. Hence, $y \in S_{y}$.

We suppose that $s_{y} \in S_{y}$. Since $\left|S_{y}\right|=\gamma_{s t}(H)-1$, we deduce that $s_{y}^{\prime} \notin S_{y}$. So, the set $D=$ $\left(S_{y} \backslash\{y\}\right) \cup\left\{s_{y}^{\prime}\right\}$ is a secure total dominating set of $H_{y}-\{y\}$ of cardinality $|D|=\left|S_{y}\right|=\gamma_{s t}(H)-1<$ $\gamma_{s t}(H-\{v\})=\gamma_{s t}\left(H_{y}-\{y\}\right.$, which is a contradiction. Hence, $s_{y} \notin S_{y}$, and so $s_{y}^{\prime} \in S_{y}$.

Let $W \subseteq V\left(G \circ \circ_{v} H\right)$ such that $W_{x}$ is induced by $S_{y}$, for any $x \in V(G)$. Since $\delta(G) \geq 2$, we deduce that $W$ is a secure total dominating set of $G \circ_{v} H$, and, as a result, $\gamma_{s t}\left(G \circ_{v} H\right) \leq|W|=\mathrm{n}(G)\left|S_{y}\right|=$ $\mathrm{n}(G)\left(\gamma_{s t}(H)-1\right)$. By Theorem 10 we obtain that $\gamma_{s t}(G \circ v)=\mathrm{n}(G)\left(\gamma_{s t}(H)-1\right)$, which completes the proof.

Theorem 12. Let $G$ be a graph with $\delta(G) \geq 2$ and $H$ a graph with no isolated vertex. Let $v \in \mathcal{L}_{w}(H)$, $N(v)=\{s\}$ and $|N(s)|=2$. If $\gamma_{s t}(H-\{v\})=\gamma_{s t}(H)-1$, then

$$
\gamma_{s t}\left(G \circ_{v} H\right) \in\left\{\mathrm{n}(G)\left(\gamma_{s t}(H)-1\right), \gamma(G)+\mathrm{n}(G)\left(\gamma_{s t}(H)-1\right)\right\} .
$$

Proof. By Theorem 10 we have that $\gamma_{s t}(G \circ v) \geq \mathrm{n}(G)\left(\gamma_{s t}(H)-1\right)$. Since $s \in \mathcal{L}(H-\{v\})$, any $\gamma_{s t}(H-\{v\})$-set $D$ contains $N[s] \backslash\{v\}$ as a subset. Let $W \subseteq V(G \circ v) \backslash V(G)$ such that $W_{x}$ is induced by $D$ for every vertex $x \in V(G)$. As for any $\gamma(G)$-set $X$, the set $X \cup W$ is a secure total dominating set of $G \circ_{v} H$, we deduce that $\gamma_{s t}\left(G \circ_{v} H\right) \leq|X \cup W|=\gamma(G)+\mathrm{n}(G) \gamma_{s t}(H-\{v\})=$ $\gamma(G)+\mathrm{n}(G)\left(\gamma_{s t}(H)-1\right)$.

Let $S$ be a $\gamma_{s t}\left(G \circ_{v} H\right)$-set such that $|S|>\mathrm{n}(G)\left(\gamma_{s t}(H)-1\right)$. For any vertex $x \in V(G)$, let $\left\{s_{x}\right\}=$ $N(x) \cap V\left(H_{x}\right)$. By Theorem 10, we can conclude that the set $Z=\left\{z \in V(G):\left|S_{z}\right|=\gamma_{s t}(H)\right\}$ is not empty. Since there exists a $\gamma_{s t}(H)$-set containing $N[s]$, we can assume, without loss of generality, that $N\left[s_{z}\right] \subseteq S_{z}$ for every vertex $z \in Z$. We claim that $Z$ is a dominating set of $G$. Let $x^{\prime} \in V(G) \backslash Z$ and suppose that $x^{\prime} \in S$. In such a case, $\left|S_{x^{\prime}}\right|=\gamma_{s t}(H)-1$ and we can define a set $W^{\prime} \subseteq V\left(G \circ_{v} H\right)$ such that $W_{x}^{\prime}$ is induced by $S_{x^{\prime}}$ for every vertex $x \in V(G)$. Notice that $W$ is a secure total dominating set of $G \circ_{v} H$ and $|W|=\mathrm{n}(G)\left(\gamma_{s t}(H)-1\right)$, which is a contradiction. Thus, $(V(G) \backslash Z) \cap S=\varnothing$, which implies that $Z$ is a dominating set of $G$ and so $\gamma_{s t}\left(G \circ_{v} H\right)=|S| \geq\left|\cup_{x \in V(G)} S_{x}\right|=|Z|+$ $\mathrm{n}(G)\left(\gamma_{s t}(H)-1\right) \geq \gamma(G)+\mathrm{n}(G)\left(\gamma_{s t}(H)-1\right)$, which completes the proof.

Theorem 13. Let $G$ be a graph such that $\delta(G) \geq 2$ and $H$ a graph with no isolated vertex. If $v \in \mathcal{L}_{w}(H)$, $N(v)=\{s\},|N(s)|=2$ and $N(s) \cap \mathcal{S}(H) \neq \varnothing$, then

$$
\gamma_{s t}(G \circ v)=\mathrm{n}(G)\left(\gamma_{s t}(H)-1\right) .
$$


Proof. For any vertex $x \in V(G)$, let $\left\{s_{x}\right\}=N(x) \cap V\left(H_{x}\right)$ and notice that any $\gamma_{s t}\left(H_{x}\right)$-set $D_{x}$ satisfies that $N\left[s_{x}\right] \subseteq D_{x}$ and $D_{x} \backslash\left\{x, s_{x}\right\}$ is a secure total dominating set of $H_{x}-\left\{x, s_{x}\right\}$. Since $\delta(G) \geq 2$, we have that $D=\bigcup_{x \in V(G)}\left(D_{x} \backslash\left\{s_{x}\right\}\right)$ is a secure total dominating set of $G \circ_{v} H$. Hence, $\gamma_{s t}\left(G \circ_{v}\right.$ $H) \leq|D|=\mathrm{n}(G)\left(\gamma_{s t}(H)-1\right)$. By Theorem 10 we obtain that $\gamma_{s t}\left(G \circ_{v} H\right)=\mathrm{n}(G)\left(\gamma_{s t}(H)-1\right)$, which completes the proof.

\section{Concluding Remarks}

It is well-known that the problem of finding the secure total domination number of a graph is NP-hard. This suggests the challenge of finding closed formulas or giving tight bounds for this parameter. In this paper we develop the theory for the class of rooted product graph. The study shows that if the root vertex is strong leaf, a support, or a universal vertex, then there exists a formula for the secure total domination number of the rooted product graph. In the remaining cases, two different behaviours are observed depending on whether the root vertex is a weak leaf or not. Although in a different way, in both cases we were able to give the intervals to which the parameter belongs. The endpoints of these intervals are expressed in terms of other domination parameters of the graphs $G$ and $H$ involved in the product, which allows us to obtain closed formulas when certain conditions are imposed on $G$ or $H$.

Author Contributions: All authors contributed equally to this work. All authors have read and agreed to the published version of the manuscript.

Funding: This research received no external funding.

Conflicts of Interest: The authors declare no conflict of interest.

\section{References}

1. Cockayne, E.J.; Grobler, P.J.P.; Gründlingh, W.R.; Munganga, J.; Van Vuuren, J.H. Protection of a graph. Util.Math. 2005, 67, 19-32.

2. Benecke, S.; Cockayne,E.J.; Mynhardt, C.M. Secure total domination in graphs. Util. Math. 2007, 74, $247-259$.

3. Cabrera Martínez, A.; Montejano, L.P.; Rodríguez-Velázquez, J.A. On the secure total domination number of graphs. Symmetry 2019, 11, 1165. [CrossRef]

4. Cabrera Martínez, A.; Montejano, L.P.; Rodríguez-Velázquez, J.A. Total weak Roman domination in graphs. Symmetry 2019, 11, 831. [CrossRef]

5. Henning, M.A.; Hedetniemi, S.T. Defending the Roman Empire-A new strategy. Discrete Math. 2003, 266, 239-251. [CrossRef]

6. Cockayne, E.J.; Dreyer, P.A., Jr.; Hedetniemi, S.M.; Hedetniemi, S.T. Roman domination in graphs. Discrete Math. 2004, 278, 11-22. [CrossRef]

7. Stewart, I. Defend the Roman Empire!. Scientific American: New York, NY, USA, 1999; Volume 281, pp. 136-138.

8. Haynes, T.W.; Hedetniemi, S.T.; Slater, P.J. Domination in Graphs: Advanced Topics.; Chapman and Hall/CRC Pure and Applied Mathematics Series; Marcel Dekker, Inc.: New York, NY, USA, 1998.

9. Haynes, T.W.; Hedetniemi, S.T.; Slater, P.J. Fundamentals of Domination in Graphs; Chapman and Hall/CRC Pure and Applied Mathematics Series; Marcel Dekker, Inc.: New York, NY, USA, 1998.

10. Henning, M.; Yeo, A. Total Domination in Graphs. Springer Monographs in Mathematics; Springer: New York, NY, USA, 2013.

11. Chellali, M.; Haynes, T.W.; Hedetniemi, S.T.; McRae, A.A. Roman 2-domination. Discrete Appl. Math. 2016, 204, 22-28. [CrossRef]

12. Boumediene Merouane, H.; Chellali, M. On secure domination in graphs. Inform. Process. Lett. 2015, 115, 786-790. [CrossRef]

13. Burger, A.P.; Henning, M.A.; Van Vuuren, J.H. Vertex covers and secure domination in graphs. Quaest. Math. 2008, 31, 163-171. [CrossRef]

14. Chellali, M.; Haynes, T.W.; Hedetniemi, S.T. Bounds on weak Roman and 2-rainbow domination numbers. Discrete Appl. Math. 2014, 178, 27-32. [CrossRef]

15. Cockayne, E.J.; Favaron, O.; Mynhardt, C.M. Secure domination, weak Roman domination and forbidden subgraphs, Bull. Inst. Combin. Appl. 2003, 39, 87-100. 
16. Klostermeyer, W.F.; Mynhardt, C.M. Secure domination and secure total domination in graphs. Discuss. Math. Graph Theory. 2008, 28, 267-284. [CrossRef]

17. Valveny, M.; Rodríguez-Velázquez, J.A. Protection of graphs with emphasis on Cartesian product graphs. Filomat 2019, 33, 319-333. [CrossRef]

18. Duginov, O. Secure total domination in graphs: Bounds and complexity. Discrete Appl. Math. 2017, 222, 97-108. [CrossRef]

19. Kulli, V.R.; Chaluvaraju, B.; Kumara, M. Graphs with equal secure total domination and inverse secure total domination numbers. J. Inf. Optim. Sci. 2018, 39, 467-473. [CrossRef]

(c) 2020 by the authors. Licensee MDPI, Basel, Switzerland. This article is an open access article distributed under the terms and conditions of the Creative Commons Attribution (CC BY) license (http:/ / creativecommons.org/licenses/by/4.0/). 